so far Bioorganic and Medicinal Chemistry has significantly fewer papers and is published less frequently (monthly as opposed to twice monthly), although the costs of both journals are similar.

In summary, Bioorganic and Medicinal Chemistry has not yet displaced the Journal of Medicinal Chemistry as the gold standard, but it is certainly worthy of serious consideration as an addition to any library subscribing to the latter.

David C. Rees is at Organon Laboratories, Newhouse, Lanarkshire ML1 5SH, UK.

\section{In fine mettle}

Carl C. Koch

Intermetallics. Editors, R. W. Cahn, C. T. Liu, G. Sauthoff, M. Yamaguchi. Elsevier. 4/yr. £219, \$335.

INTERMETALLICS - ordered compounds composed of two or more metals - have generated interest in both scientific and engineering circles for many years owing to their often unique properties. More than a decade ago, several 'breakthroughs' were reported in the ability to make these normally very brittle materials ductile. This led to a great resurgence in research on intermetallics as structural materials with possible applications at elevated temperatures. Today, intermetallic compounds are probably the most studied class of metallic materials. Research on intermetallics is published throughout the range of journals on materials science and condensed-matter physics as well as in large symposia proceedings.

At four issues a year, Intermetallics can hope to publish only a small proportion of this extensive literature. But the editors are in the enviable position of being able to limit their journal to papers of the highest quality. Review articles, research articles, short communications and comments on papers are all solicited.

The production quality is also excellent. This is important for materials science journals, where clear and accurate photographs (such as transmission electron micrographs) are often critical to the interpretation of experimental results.

The journal should be attractive for authors: there are no page charges and 25 reprints are supplied free. The subscription price unfortunately puts the journal out of reach of most individual subscribers, and given the tight budgets of libraries, it may also prevent many institutions from access to what is an important new journal in a vital field.

Carl C. Koch is in the Department of Materials Science and Engineering, North Carolina State University, Box 7907, Raleigh, North Carolina 27695-7907, USA.

\title{
Fullerene fever
}

\section{Arthur F. Hebard}

Fullerene Science and Technology. Editor T. Braun. Dekker. 4/yr. \$250 (institutional); $\$ 125$ (personal).

IN the foreword to the first issue of this new journal, the editor opens with the statement that "some facts indicate that fullerene fever is more than just a fashion". He then points out that the general fascination with these beautiful molecules and well established but broadly dispersed research activity justify the need for a dedicated journal that will "provide an interdisciplinary and cross-disciplinary platform for international communication".

One might justifiably ask, however, whether the cure for fullerene fever is the birth of a new journal. It is not clear whether fullerene science will develop a tradition of its own or become assimilated into the more general field of carbon science. For this new journal to be successful, it will therefore have to create a unique niche and cater for a multidisciplinary readership by attracting seminal contributions from a broad spectrum of researchers. This is a daunting task

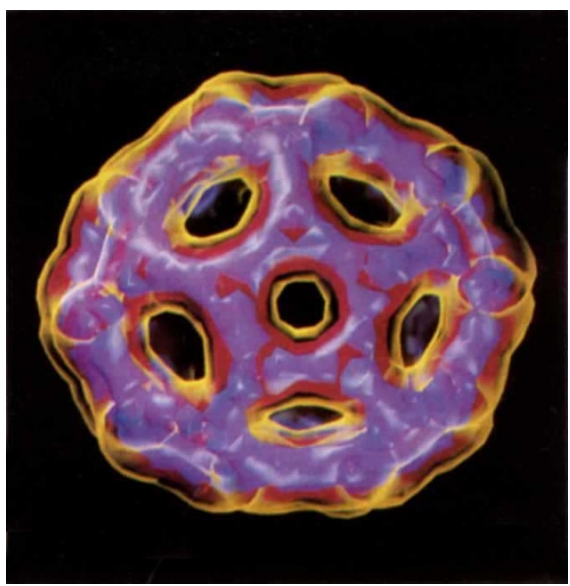

Electron-distribution simulation of fullerene.

because many potential contributors will be tempted to submit to the numerous established journals that already have a wide readership and which often have sections or special issues dedicated to fullerenes.

Fullerene Science and Technology does have a highly qualified multinational editorial board, which should help in the soliciting of papers. Five of the editors have shown their commitment by writing or co-writing articles in the inaugural issue. Two of the four volumes reviewed are proceedings issues devoted to the Fourth and Fifth General Symposiums on Fullerene Science and Technology held in Tokyo, Japan. Unfortunately, the reduction by more than half in the number of contributions to successive symposium issues may indicate lagging interest - a portent of trouble for a struggling new journal.

The scope of this journal at its incubation stage is determined by the papers submitted. Although in general the quality of work reported is fairly high, a few of the manuscripts seem rather preliminary and inconsequential. A more varied format that included brief communications, comments, guest editorials, and technical notes might increase the appeal of the journal.

Arthur F. Hebard is at AT\&T Bell Laboratories, 600 Mountain Avenue, Murray Hill, New Jersey 07974, USA.

\section{Looking good}

\author{
Ian A. Walmsley
}

Pure and Applied Optics. Editor M. Bertolotti. Institute of Physics/American Institute of Physics. 6/yr. USA, Canada and Mexico $\$ 272$, elsewhere $£ 132$.

THIs journal, published on behalf of the European Optical Society as Part A of a two-part set, covers just about all of classical optics: that is, those optical phenoma के that do not require a description in terms of quantum fields. So nonlinear optics, laser physics and spectroscopy are included. In fact, topics under the heading of quantum optics are specifically excluded, presumably coming under the jurisdiction of Part B.

Although the reasoning behind the separation is clear (and notably different from that behind the splitting of the Journal of the Optical Society of America in 1984), it sometimes leads to a strange juxtaposition of articles; one on caustics in ray propagation, for example, sandwiched between papers on phase conjugation and mode-locked laser theory.

The editorial board is large, and its members are all eminent scientists and engineers, giving the journal a fairly international perspective and placing it firmly in the ranks of other distinguished titles. Both full-length papers and letters (of specified maximum length) are published, and there are also book reviews and lengthier review articles.

An attractive feature for authors is the absence of page charges (and this even with a fairly modest library subscription rate), and, as a further nice touch, a few off-prints are provided gratis by the publisher. The journal will be an important addition to any comprehensive optics research library.

Ian A. Walmsley is in the Institute of Optics, University of Rochester, Rochester, New York 14627, USA. 